\title{
Microperçage de polymères par laser à excimères
}

\author{
J. Lopez et S. Lazare
}

\author{
Laboratoire de Physico-Chimie Moléculaire, UMR 5803 du CNRS, Université de Bordeaux I, \\ 351 cours de la Libération, 33405 Talence, France
}

\begin{abstract}
Résumé : Le microperçage par laser UV nécessite un faisceau intense et de faible ouverture. Il n'introduit pas de détérioration thermique ou mécanique de la cible. Dans certaines conditions, il est possible d'obtenir des trous filiformes, reproductibles et à hauts rapports de forme $(\Phi / \mathrm{d} \approx 600)$ sur de nombreux matériaux. De manière générale, plus un polymère est absorbant à $248 \mathrm{~nm}$, meilleure est la résolution et plus il est facile d'obtenir des trous profonds, mais plus la vitesse de perçage est faible. Ces résultats prometteurs laissent entrevoir un large champ d'applications dans le domaine du micro-usinage.
\end{abstract}

\section{INTRODUCTION}

L'intérêt majeur de la photoablation de polymères par rayonnement laser ultraviolet réside dans le fait qu'elle met en jeu une interaction non-thermique (processus photochimique, faible durée d'impulsion) à une résolution submicronique. Potentiellement, les applications industrielles sont nombreuses : gravure, microperçage, dépôt, modification de surface, décapages, etc.. Par ailleurs, l'expulsion à grande vitesse de fragments d'ablation pendant l'interaction laser-matière permet d'évacuer l'excès d'énergie sous forme d'énergie cinétique. Les détériorations thermiques et mécaniques de la cible sont donc généralement négligeables.

Les travaux publiés [1,2] ont montré les performances des lasers UV pulsés dans le domaine du microperçage. Ce travail $[3,4]$ révèle de nouveau qu'il est possible d'atteindre des rapports de forme exceptionnellement importants $(\Phi / \mathrm{d} \approx 600)$. Les conditions d'obtention de tels rapports sont succinctement présentés dans la suite.

\section{SYSTEME DE PROJECTION (figure 1)}

Le principe du système développé consiste à faire l'image du masque sur la surface de la cible par l'intermédiaire d'une lentille de courte focale. Son intérêt majeur réside dans la possibilité de mettre en forme des faisceaux intenses (fluence $\left.>10 \mathrm{~J} / \mathrm{cm}^{2}\right)$ de faible ouverture $\left(10-15^{\circ}\right)$ et de faible diamètre $(2$ à $100 \mu \mathrm{m}$ ). La mesure de tels faisceaux est particulièrement difficile à cause de leur faible diamètre et de leur intensité. Par contre, l'observation du perçage renseigne immédiatement sur la qualité du faisceau, et en pratique permet sa mise au point. Le laser à excimères utilisé est un Lambda Physik LPX220i (248 nm,

$25 \mathrm{~ns}$, div. 1-3 mrad, $300 \mathrm{~mJ} /$ impulsion)

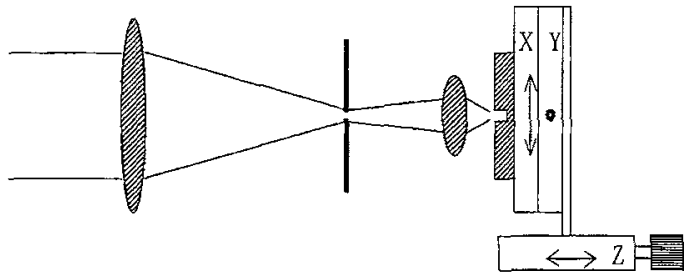

Figure 1. Système de projection : taux de réduction 5 , focale de la lentille de projection $25 \mathrm{~mm}$, fluence sur la cible 0,5 à 50 $\mathrm{J} / \mathrm{cm}^{2}$, masque Mo diamètre 5 à $500 \mu \mathrm{m}$, table $\mathrm{Z}$ mise au point, table $X Y$ prog. pour centrage et usinage (résolution $0,1 \mu \mathrm{m}$ ).

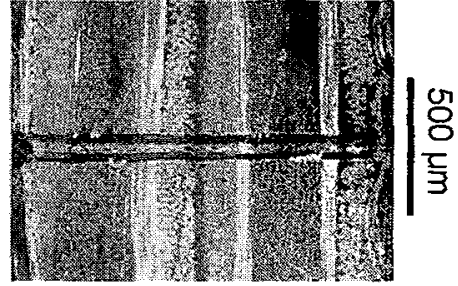

Figure 2. Matériau composite micropercé à $248 \mathrm{~nm}$ en 2000 impulsions à $3 \mathrm{~J} / \mathrm{cm}^{2}$, diamètre $100 \mu \mathrm{m}$, longueur $1,5 \mathrm{~mm}$ (micrographie obtenue après enrobage, découpe et polissage) [5]. 
La mâttrise des paramètres expérimentaux a permis l'obtention de trous filiformes, reproductibles, et à hauts rapports de forme sur un grand nombre de matériaux (figures 2 et 3 ). D'une manière générale, le rapport de forme dépend d'un ensemble de phénomènes liés, soit à la qualité du faisceau (longueur d'onde, divergence, intensité), soit aux caractéristiques de l'interaction laser-matière (seuil d'ablation, nature de l'interaction, mode de décomposition de la cible). La résolution de l'usinage laser est limitée par la mise en forme du faisceau (optique) et par la nature de la cible (zone affectée). La position optimale de la cible correspond au plan conjugué image du masque. Pour des masques inférieurs à $10 \mu \mathrm{m}$, les effets de diffraction produisent un étalement du spot sur la cible. La chute d'intensité qui en découle rend les perçages profonds irréalisables.

(a)

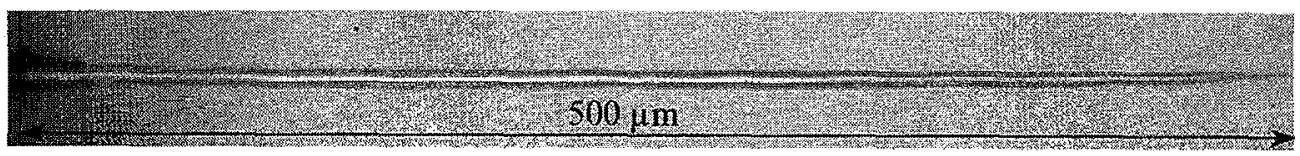

(b)

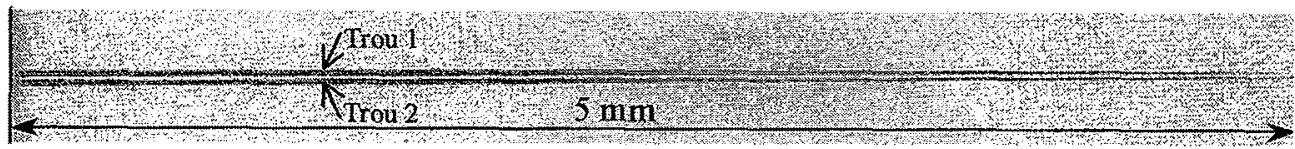

(c)

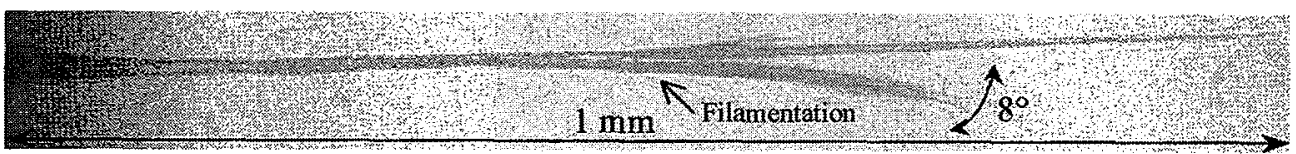

Figure 3. Micrographies optiques de trous réalisés à $248 \mathrm{~nm}$ (a) PMMA trou de diamètre $8 \mu \mathrm{m}$, longueur $500 \mu \mathrm{m}$ (b) PET, 2 trous adjacents de diamètre $30 \mu \mathrm{m}$ et longueur $5 \mathrm{~mm}$, distants de moins de $10 \mu \mathrm{m}$ l'un de l'autre (c) PC, trou de diamètre environ $20 \mu \mathrm{m}$ et longueur $1 \mathrm{~mm}$, avec un filament incliné de $8^{\circ}$ par rapport à l'axe principal du trou. Le phénomène de filamentation (montré ici à titre d'exemple) n'apparait que dans des conditions bien précises.

\section{ABLATION PROFONDE DE MATERIAUX MODELES}

L'observation au microscope optique du site d'irradiation permet de suivre le perçage de matériaux transparents en temps réel, et en particulier d'observer la transition ablation de surface-ablation profonde. En début de perçage, la vitesse est fonction de la fluence [6], mais progressivement elle tend vers une valeur constante caractéristique du matériau et indépendante de la fluence (figure 4, tableau 1). Ce phénomène de saturation résulte du découplage laser-cible provoqué par l'apparition d'un plasma absorbant au fond du trou dès les premiers instants de l'interaction. L'utilisation de fortes fluences ( $>5$ $\mathrm{J} / \mathrm{cm}^{2}$ ) et le confinement de l'interaction par les parois du trou augmentent les collisions au sein du panache, et privilégient ainsi l'apparition d'un plasma à des fluences sensiblement inférieures à celles nécessaires en ablation de surface.
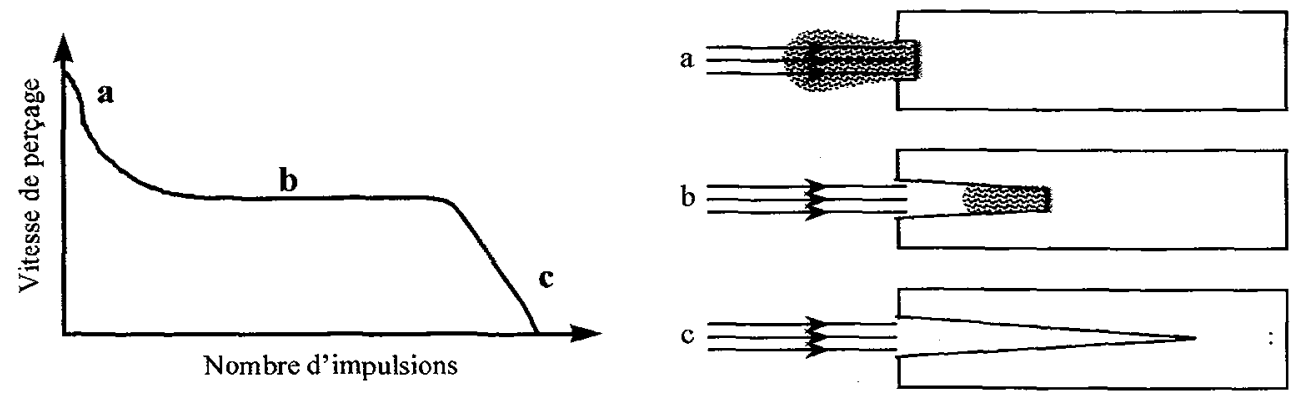

Figure 4. Evolution de la vitesse de perçage avec le nombre d'impulsions (a) Ablation de surface, la vitesse dépend de la fluence (b) Ablation profonde, les parois du trou interagissent avec le panache, la vitesse est constante, environ $1 \mu \mathrm{m}$ par impulsion (c) Arrêt spontané du perçage par manque d'énergie en fond de trou. 
Tableau 1. Caractéristiques d'ablation et de perçage de différents polymères

$\begin{array}{ccccccc} & \text { PMMA } & \text { PI } & \text { PC } & \text { PET } & \text { PS } & \text { PEEK } \\ \text { Profondeur de peau à } 248 \mathrm{~nm} & 150 \mu \mathrm{m} & 0,045 \mu \mathrm{m} & 1,0 \mu \mathrm{m} & 0,065 \mu \mathrm{m} & 1,6 \mu \mathrm{m} & - \\ \text { Seuil d'ablation }\left(\mathrm{m} J / \mathrm{cm}^{2}\right) & 250 & 54 & 40 & 30 & 40 & 50 \\ \text { Vit. perçage }(\mu \mathrm{m} / \text { impulsion }) & 2,5 & 0,4 & 0,8 & 0,7 & - & 0,6 \\ \text { Rapport de forme max Rm } & 255 & 360 & 390 & 565 & 315 & 385 \\ \text { Fluence } \mathrm{Fc}\left(\mathrm{J} / \mathrm{cm}^{2}\right) & 5,0 & 2,0 & 3,3 & 1,8 & 4,5 & 3,0\end{array}$

Une partie de l'énergie laser se propage jusqu'au fond du trou en direct. Néanmoins, la longueur des trous (parfois $1 \mathrm{~cm}$ ) est largement supérieure à la profondeur de champ du système de projection (environ $100 \mu \mathrm{m})$. Par ailleurs, la présence de filaments hors de l'axe principal du trou prouve indéniablement l'existence de réflexions multiples sur les parois internes du trou (figure 3c). Un trou se comporte donc comme un guide d'onde, ce qui facilite l'obtention de grand rapport de forme (figure 4).

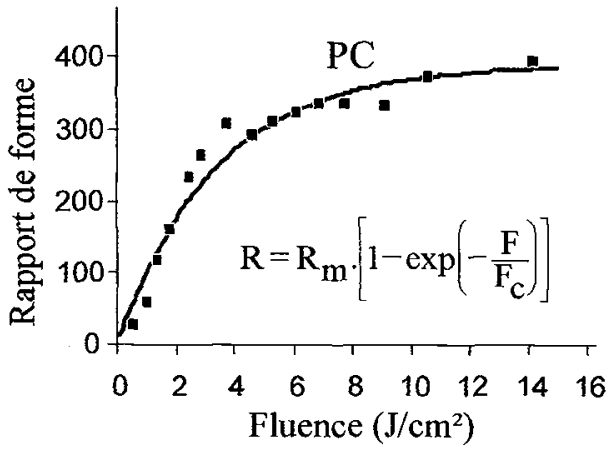

Figure 5. Evolution du rapport de forme avec la fluence: Les 2 paramètres indépendants $\mathrm{Fc}$ et $\mathrm{Rm}$ sont caractéristiques du polymère (tableau 1).

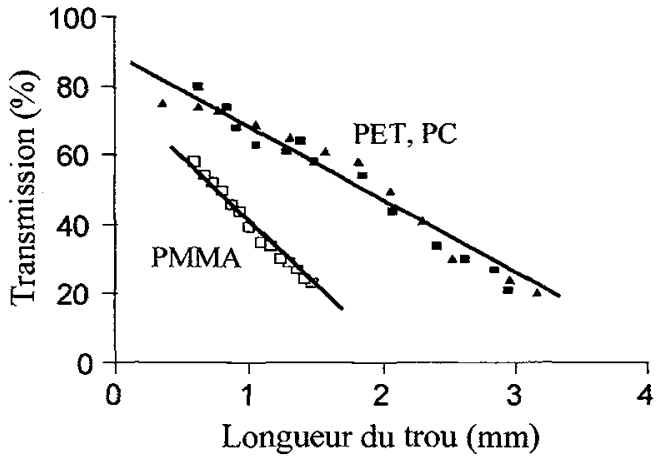

Figure 6. Transmission énergétique du trou (rapport énergie en sortie / énergie incidente) en fonction de sa longueur.

La mesure de l'énergie en sortie de trou indique une décroissance linéaire de l'énergie transmise avec la profondeur (figure 6). Il y a donc absorption de l'énergie laser au niveau des parois internes du trou. Cette perte d'énergie est d'autant plus rapide que le trou est conique et que le seuil d'ablation du matériau est élevé. Elle justifie l'arrêt spontané du perçage au-delà d'une certaine profondeur. Dans le cas du PMMA, la perte d'énergie est de $4 \%$ par tronçon de trou de 100 microns. Pour un trou de diamètre $50 \mu \mathrm{m}$ et longueur $1 \mathrm{~mm}, 20 \%$ de l'énergie sont stoppés en surface, $40 \%$ sont absorbés sur les parois internes du trou $\left(30 \mathrm{~mJ} / \mathrm{cm}^{2}\right)$, et $40 \%$ sont transmis en direct ou par réflexions multiples jusqu'au fond du trou (6 $\mathrm{J} / \mathrm{cm}^{2}$ ). Le flux d'énergie au fond du trou en formation est 200 à 300 fois supérieur à celui sur les parois internes. Cela explique l'aspect unidirectionnel des trous.

Les figures 7 et 8 résument les différentes étapes de l'interaction laser-matière pour un trou en formation et pour une impulsion donnée. La vitesse moyenne de perçage donne une estimation du volume de matière expulsée. Une partie de cette matière est susceptible de se redéposer sur les parois internes et faire obstacle au développement du trou.

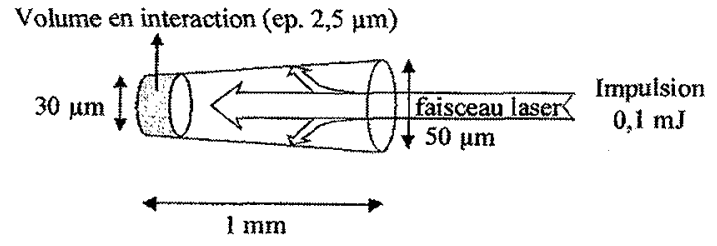

Figure 7. PHASE 1 IRRADIATION LASER: Propagation de l'impulsion jusqu'au fond du trou en direct ou par réflexions multiples, absorption du rayonnement, et ablation.

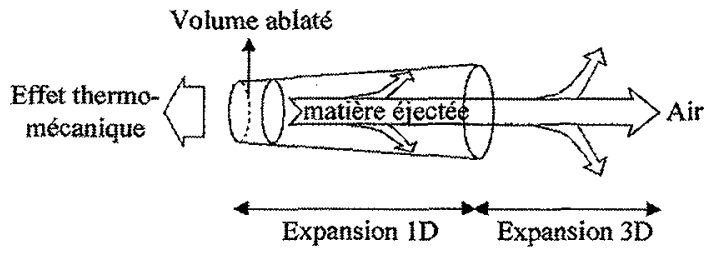

Figure 8. PHASE 2 EMISSION DU PANACHE: Expansion 1D du panache, interaction avec les parois, expansion 3D hors du trou, et redépôt éventuel sur le pourtour du trou. 
Il est possible de percer un grand nombre de matériaux (polymères, métaux, céramiques, verres, cristaux, composites, diamant, etc.), en revanche, la qualité et les performances du perçage vont dépendre de la nature de la cible. Les disparités observées proviennent des différences d'absorption et de mécanismes d'ablation.

Pour le PMMA, peu absorbant à $248 \mathrm{~nm}$, la modification induite au niveau des parois internes peut être mesurée et identifiée par Micro Spectroscopie Raman Confocale [7]. Cette technique permet de sonder la matière autour du trou avec une résolution de $1 \mu \mathrm{m}^{3}$. La modification (création d'insaturations et dépolymérisation) s'étend sur un rayon de plus de $50 \mu \mathrm{m}$ autour du trou. Cette zone affectée limite la résolution de l'usinage laser. L'échauffement du PMMA pendant l'interaction provoque l'expulsion de gouttelettes de PMMA, partiellement dépolymérisé et liquifié, de taille supérieure au micron (figure $9 b$ ). Néanmoins, le PMMA est un cas à part, car pour des polymères absorbants à $248 \mathrm{~nm}$ (PET, PI, PEEK, PC) la zone affectée autour du trou, de l'ordre du micron, ne limite pas la résolution de l'usinage laser. L'interaction est ici principalement photochimique. La matière ablatée est fortement décomposée (éjection de gaz ou de particules de taille inférieure au micron) (figure 9a).

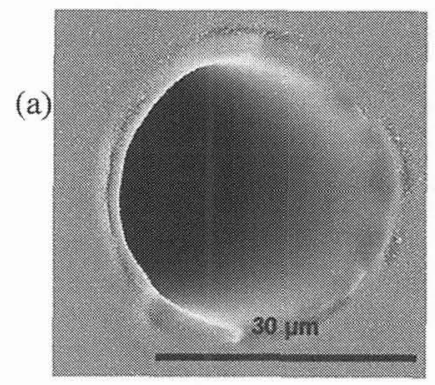

(b)

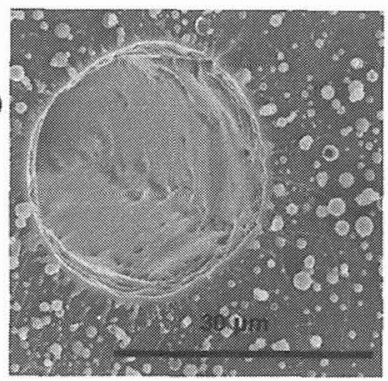

(c)

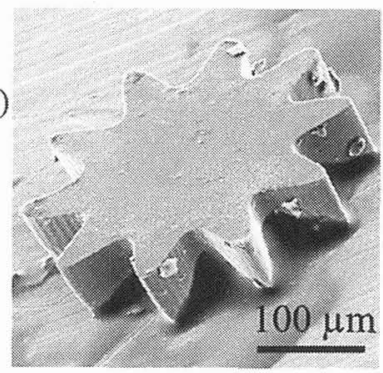

Figure 9. Micrographies MEB (a) PC trou diamètre $33 \mu \mathrm{m}$, longueur $5 \mathrm{~mm}$ (b) PMMA trou diamètre $30 \mu \mathrm{m}$, longueur $2 \mathrm{~mm}$ (c) Microfabrication : découpe laser d'une roue dentée en PET diamètre $400 \mu \mathrm{m}$, ép. $100 \mu \mathrm{m}$ (François Weisbuch).

\section{CONCLUSION}

Le microperçage par laser UV nécessite un faisceau intense et de faible ouverture. Il n'introduit pas de détérioration thermique ou mécanique de la cible. D’une manière générale, plus un polymère sera absorbant à $248 \mathrm{~nm}$, meilleure sera la résolution et plus il sera facile d'obtenir des rapports de forme élevés $(>300)$, mais plus la vitesse de perçage sera faible. Ces résultats particulièrement prometteurs laissent entrevoir un large champ d'applications dans le domaine du micro-usinage (figure 9c).

\section{Remerciements}

Région Aquitaine pour cofinancement, Pr J.M.Turlet (CPMOH) pour la Micro Spectroscopie Raman Confocale, Pr A.Catherinot pour son aide.

\section{Références}

1. B.Braren et R.Srinivasan, J.Vac.Sci.Technol., B3, 3, p913-917 (1985)

2. D.Damiani, Ann.Phys., C1, n5, 19, p261-268 (1994); thèse Université de Limoges (1991)

3. J.Lopez, Thèse de l'Université de Bordeaux (1997)

4. S.Lazare, D.Drilhole, J.Lopez, F.Weisbuch, Le vide : science, technique et applications, 287, Sept.Oct.Nov. (1998)

5. C.Sourdet, D.Ordièra (Dassault Aviation Biarritz), S.Lazare et J.Lopez (LPCM), Rapport PAMM sur le partenariat recherche/industrie en Aquitaine (1996)

6. S.Lazare et V.Granier, Laser Chem., 10, p25-40 (1988)

7. S.Lazare, J.Lopez, J.M.Turlet, S.Kufner, M.Kufner et P.Chavel, Appl.Opt., 35, 22 (1996) 